\title{
Semivector Finite-Difference Formula for the Analysis of a Step-Index Waveguide With a Tilted Interface
}

\author{
Junji Yamauchi, Senior Member, IEEE, Seiji Harada, Shingo Kobori, and Hisamatsu Nakano, Fellow, IEEE
}

\begin{abstract}
A three-point finite-difference formula is derived for the analysis of a step-index waveguide with a tilted interface. The formula is suitable for the use of the alternating-direction implicit method. Through the analysis of a rib waveguide with a sloped side wall and a hollow triangular waveguide, improvement in accuracy and stability is demonstrated.
\end{abstract}

Index Terms-Alternating-direction implicit method (ADIM), beam-propagation method (BPM), finite-difference (FD) formula.

\section{INTRODUCTION}

$\mathbf{C}$ ONSIDERABLE effort has been made to improve a finite-difference (FD) formula for the analysis of a step-index optical waveguide [1]-[4]. Pioneering work on a modified FD formula was carried out by Stern [1], who introduced the concept of a semivectorial mode. This approach assumes that all the discontinuities of dielectric materials are either parallel or orthogonal to $x$ - and $y$-axes, and that the fields are either $x$ - or $y$-polarized. It should be noted, however, that the situation becomes more complicated when a tilted interface is treated. Xia and Yu have proposed a scheme for the analysis of a step-index waveguide with a tilted interface [5], [6]. Although their scheme is significantly effective in terms of accuracy and stability, the scheme requires the field evaluation at seven mesh points in the transverse plane ("seven-point scheme"). This unfortunately prohibits the use of the efficient algorithm, such as the alternating-direction implicit method (ADIM), resulting in low computational efficiency.

To overcome this problem, the authors have proposed a simple FD formula for the analysis of a step-index waveguide with a tilted interface [7], but only the formula for the electric field has been presented. The purpose of this letter is to develop a three-point FD formula, which can be used for the analysis of both electric and magnetic semivector fields. The developed formula is suitable for the use together with the ADIM where a five-point scheme is required. To test the validity of the present formula, we simulate a rib waveguide with a sloped sidewall

Manuscript received September 11, 2009; revised October 06, 2009. First published October 23, 2009; current version published December 01, 2009. This work was supported in part by MEXT, Grant-in-Aid for Scientific Research (c) (19560355).

J. Yamauchi, S. Harada, and H. Nakano are with the Faculty of Engineering, Hosei University, Tokyo 184-8584, Japan (e-mail: j.yma@k.hosei.ac.jp; seiji. harada.bp@gs-eng.hosei.ac.jp; hymat@hosei.ac.jp).

S. Kobori is with Advanced Technology R\&D Center, Mitsubishi Electric Corporation, Hyogo 661-8661, Japan (e-mail: Kobori.Shingo@cj.MitsubishiElectric.co.jp).

Color versions of one or more of the figures in this letter are available online at http://ieeexplore.ieee.org.

Digital Object Identifier 10.1109/LPT.2009.2034751

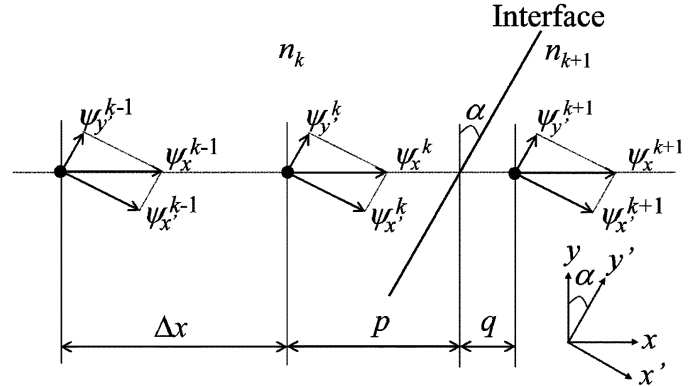

Fig. 1. Decomposition of field components near a tilted interface.

and a triangular hollow waveguide using the beam-propagation method (BPM).

\section{FORMULATION}

Consideration is given to the evaluation of the field derivative with the discontinuity at the tilted interface shown in Fig. 1. The axes normal and tangential to the tilted interface are defined as $x^{\prime}$ and $y^{\prime}$, respectively. In this letter, we discuss the treatment of the semivector field $\psi_{x}$, which can be decomposed into $\psi_{x^{\prime}}$ and $\psi_{y^{\prime}}$. The relation among $\psi_{x}, \psi_{x^{\prime}}$, and $\psi_{y^{\prime}}$ requires that $\psi_{x}=$ $\psi_{x^{\prime}} \cos \alpha+\psi_{y^{\prime}} \sin \alpha$, so that we get

$$
\frac{\partial^{2} \psi_{x}}{\partial x^{2}}=\frac{\partial^{2} \psi_{x^{\prime}}}{\partial x^{2}} \cos \alpha+\frac{\partial^{2} \psi_{y^{\prime}}}{\partial x^{2}} \sin \alpha .
$$

The second derivative is evaluated by the modified FD formula developed in [2]. Taking into account the boundary condition of the field components, we substitute the formula for the component normal to the interface into $\partial^{2} \psi_{x^{\prime}} / \partial x^{2}$, while that for the tangential component into $\partial^{2} \psi_{y^{\prime}} / \partial x^{2}$. Since the relations among $\psi_{x}, \psi_{x^{\prime}}$, and $\psi_{y^{\prime}}$ are given by $\psi_{x^{\prime}}=\psi_{x} \cos \alpha$ and $\psi_{y^{\prime}}=\psi_{x} \sin \alpha, \partial^{2} \psi_{x} / \partial x^{2}$ can be written as

$$
\begin{aligned}
\frac{\partial^{2} \psi_{x}}{\partial x^{2}}= & \left(a_{2}^{n} \cos ^{2} \alpha+a_{2}^{t} \sin ^{2} \alpha\right) \psi_{x}^{k-1} \\
& +\left(b_{2}^{n} \cos ^{2} \alpha+b_{2}^{t} \sin ^{2} \alpha\right) \psi_{x}^{k} \\
& +\left(c_{2}^{n} \cos ^{2} \alpha+c_{2}^{t} \sin ^{2} \alpha\right) \psi_{x}^{k+1}
\end{aligned}
$$

Note that the superscripts $n$ and $t$, respectively, means the normal and tangential components. The coefficients, $a_{2}, b_{2}$, and $c_{2}$ are defined as follows:

$$
\begin{aligned}
& a_{2}=f_{1} /\left(\Delta x^{2} f_{1} / 2+f_{2} \Delta x\right) \\
& b_{2}=-\left(f_{0} \Delta x+f_{1}\right) /\left(\Delta x^{2} f_{1} / 2+f_{2} \Delta x\right) \\
& c_{2}=1 /\left(\Delta x f_{1} / 2+f_{2}\right)
\end{aligned}
$$


TABLE I

VALUES OF $\theta_{E}$ AND $\theta_{H}$

\begin{tabular}{|c|c|c|c|c|}
\hline \multirow{2}{*}{} & \multicolumn{2}{|c|}{ Electric field } & \multicolumn{2}{c|}{ Magnetic field } \\
\cline { 2 - 5 } & Tangential & Normal & Tangential & Normal \\
\hline$\theta_{E}$ & 1 & $n_{k}^{2} / n_{k+1}^{2}$ & 1 & 1 \\
\hline$\theta_{H}$ & 1 & 1 & $n_{k+1}^{2} / n_{k}^{2}$ & 1 \\
\hline
\end{tabular}

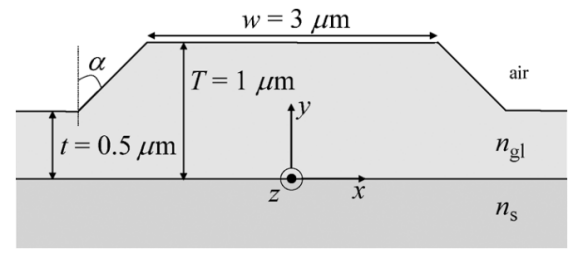

Fig. 2. Rib waveguide with a sloped sidewall.

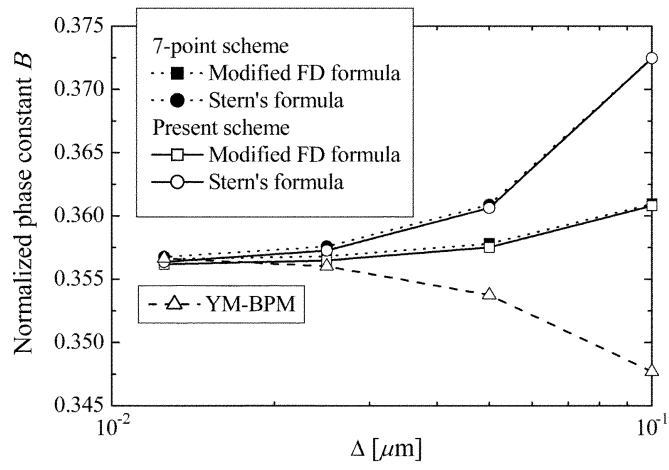

Fig. 3. Normalized phase constant for $E^{x}$-mode as a function of $\Delta$.

where

$$
\begin{aligned}
& f_{0}=\theta_{E}\left(1+q^{2} \eta / 2\right) \\
& f_{1}=\theta_{E}\left(p+p q^{2} \eta / 2\right)+\theta_{H}\left(q+q^{3} \eta / 6\right) \\
& f_{2}=\theta_{E}\left(p^{2}+q^{2}\right) / 2+\theta_{H} p q
\end{aligned}
$$

in which $\eta=k_{0}^{2}\left(n_{k}^{2}-n_{k+1}^{2}\right)$ [8], where $k_{0}$ is the free-space wavenumber. $\theta_{E}$ and $\theta_{H}$ are appropriately chosen according to Table I.

As expected, for $\alpha=0^{\circ}$, (2) reduces to the expression of the standard modified FD formula whose accuracy is second-order for $p=q$ [2], [8]. Note, however, that the accuracy of the modified FD formula becomes first-order for $p \neq q$. We, therefore, carry out the calculation under the condition of $\Delta x=\Delta y \tan \alpha$ to maintain the relation of $p=q$. It is worth mentioning that (2) is a three-point formula, and therefore, generates a five-point scheme for the ADIM, when a 3-D waveguide is treated. The expression for the $\psi_{y}$ component can be obtained similarly.

\section{DISCUSSION}

To clarify the validity of the present formula, we first carry out the eigenmode analysis of a rib waveguide with a sloped sidewall, as shown in Fig. 2. The tilt angle is taken to be $\alpha=$ $45^{\circ}$. The waveguide with $\alpha=0^{\circ}$ corresponds to that used as a classical benchmark [9]. The operating wavelength is set to be $\lambda=1.15 \mu \mathrm{m}$. No absorbing boundary condition is adopted in this analysis.

Fig. 3 shows the normalized phase constant as a function of the transverse sampling width $\Delta(=\Delta x=\Delta y)$. The $E^{x}$ [quasi-
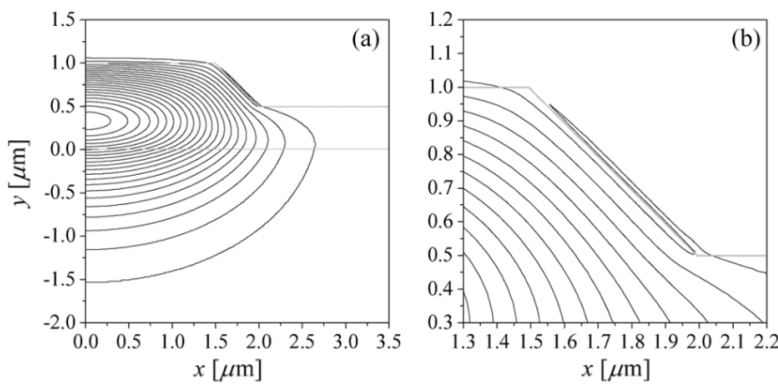

Fig. 4. Field distribution for $E^{x}$-mode. (a) Total view. (b) Expanded view.

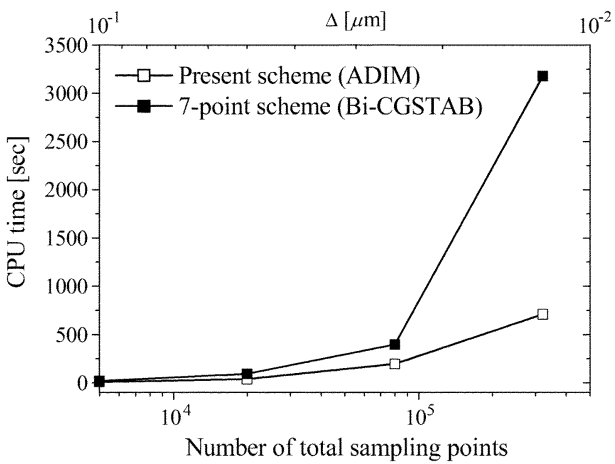

Fig. 5. CPU time required for the eigenmode analysis.

transverse-electric (TE)] mode is treated using the imaginarydistance procedure. For comparison, the data obtained with the "seven-point scheme" [5] are also presented. It is revealed that the present scheme provides almost the same results as those obtained with the "seven-point scheme," in spite of the reduced sampling points. For further reference, the data obtained with the Yee-mesh BPM [4] are plotted. Good agreement is found to exist between the converged values. Fig. 3 also shows that the present modified formula exhibits faster convergence than the Stern formula. This fact was also pointed out in [6]. A typical field distribution obtained with the $E^{x}$-mode is shown in Fig. 4. The field discontinuity across the sidewall observed for $\alpha=0^{\circ}$ tends to be smaller as $\alpha$ is increased.

Although not illustrated, we have confirmed that the phase constant obtained with the $H^{y}$-mode is identical to that with the $E^{x}$-mode. As expected, the magnetic field across the sloped sidewall is continuous in contrast to the electric field.

To show the effectiveness of the present scheme, we evaluate the CPU time, where a PC with Intel Core2 Quad processor $(2.66 \mathrm{GHz})$ is used. Fig. 5 shows a comparison between the two methods. While the "seven-point scheme" must be employed together with an iterative solver such as the Bi-CGSTAB, the present formula can be employed with the ADIM, which leads to a simpler treatment using the Thomas algorithm. As a result, the present scheme achieves higher computational efficiency than the "seven-point scheme."

We next consider the hollow isosceles triangular waveguide shown in Fig. 6. The base length is fixed to be $w=30 \mu \mathrm{m}$. The vertex angle is designated as $\phi$. The refractive indices of the core and the cladding are denoted as $n_{\mathrm{co}}$ and $n_{\mathrm{cl}}$, respectively. The core is taken to be the air region. The operating wavelength is chosen to be $\lambda=1.55 \mu \mathrm{m}$. In the analysis, $\Delta x$ is fixed to be $w / 750=0.04 \mu \mathrm{m}$, while $\Delta y$ is determined by $\Delta x / \tan (\phi / 2)$. 


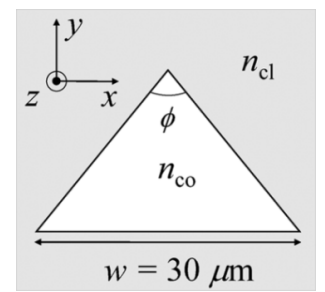

Fig. 6. Hollow triangular waveguide.

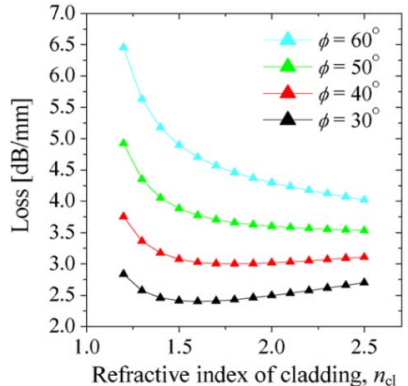

(a)

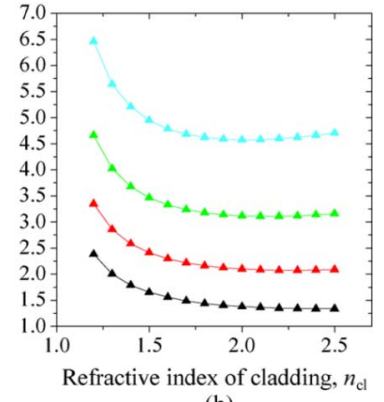

(b)
Fig. 7. Loss as a function of $n_{\mathrm{cl}}(w=30 \mu \mathrm{m})$. (a) $E^{x}$-mode. (b) $E^{y}$-mode.
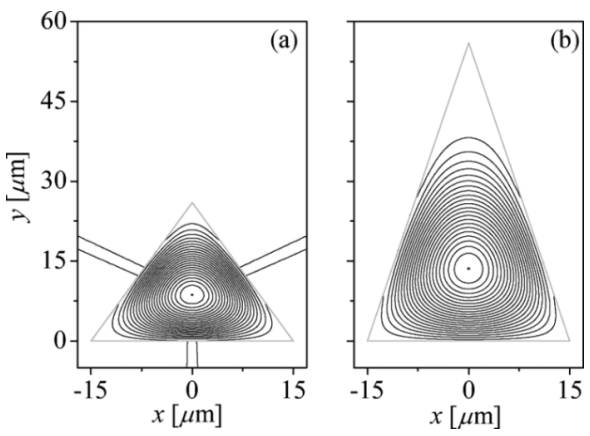

Fig. 8. Field distributions for $E^{x}$-mode $\left(n_{\mathrm{cl}}=1.45\right)$. (a) $\phi=60^{\circ}$. The lines in the cladding represent leaky waves. (b) $\phi=30^{\circ}$.

A perfectly matched layer is used as the boundary condition [10].

Figs. 7(a) and (b), respectively, show the losses of the $E^{x_{-}}$ and $E^{y}$-modes as a function of $n_{\mathrm{cl}}$ for several values of $\phi$. It is found that the loss tends to decrease as $\phi$ is decreased, since the core area becomes larger. To interpret the behavior of the loss in more detail, we should recall the loss properties in a hollow slab waveguide, which has a minimum loss condition of $n_{\mathrm{cl}}^{\min }=\sqrt{2} n_{\mathrm{co}}$ for the transverse-magnetic (TM) mode [11]. In Fig. 7(a), there exists a minimum loss for small $\phi$ 's, since the triangular waveguide operating in the $E^{x}$-mode approaches a slab waveguide operating in the TM mode as $\phi$ is decreased. Owing to the same reason, an extremum of loss appears for large $\phi$ 's for the $E^{y}$-mode [see the data for $\phi=60^{\circ}$ in Fig. 7(b)]. In other cases, the extremum of the loss disappears, since the waveguide approaches a slab waveguide operating in the TE mode. Typical field distributions for the $E^{x}$-mode are presented in Fig. 8. It is seen that the field is well confined for $\phi=30^{\circ}$.

Further calculation shows that the present formula allows us to stably simulate the propagating beam in the real-distance BPM, which is not obtainable in the conventional FD formula

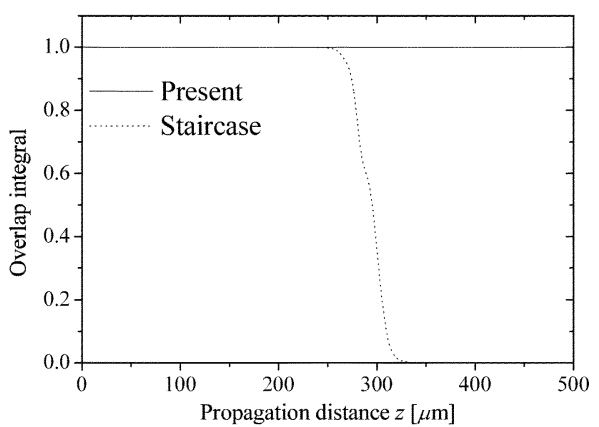

Fig. 9. Overlap integral versus propagation distance $\left(\phi=30^{\circ}, n_{\mathrm{cl}}=3.44\right)$.

with a staircase approximation. Fig. 9 shows the overlap integral observed in the real-distance BPM. The present formula improves the numerical stability over a long propagation distance.

\section{CONCLUSION}

We have derived a simple three-point FD formula for the semivector analysis of a step-index optical waveguide with a tilted interface. The present formula can be incorporated with the ADIM, yielding a high computational efficiency. A rib waveguide with a sloped sidewall and a hollow triangular waveguide are analyzed using the BPM. The high accuracy and stability are demonstrated through the analysis of the phase and attenuation constants. Extension to a fullvector analysis is yet to be researched.

\section{REFERENCES}

[1] M. S. Stern, "Semivectorial polarised finite difference method for optical waveguides with arbitrary index profiles," Proc. Inst. Elect. Eng., vol. 135, no. 1, pt. J, pp. 56-63, Feb. 1988.

[2] J. Yamauchi, M. Sekiguchi, O. Uchiyama, J. Shibayama, and H. Nakano, "Modified finite-difference formula for the analysis of semivectorial modes in step-index optical waveguides," IEEE Photon. Technol. Lett., vol. 9, no. 7, pp. 961-963, Jul. 1997.

[3] Y. P. Chiou, Y. C. Chiang, and H. C. Chang, "Improved three-point formulas considering the interface conditions in the finite-difference analysis of step-index optical devices," J. Lightw. Technol., vol. 18, no. 2, pp. 243-251, Feb. 2000

[4] T. Ando, H. Nakayama, S. Numata, J. Yamauchi, and H. Nakano, "Eigenmode analysis of optical waveguides by a Yee-mesh-based imaginary-distance propagation method for an arbitrary dielectric interface," J. Lightw. Technol., vol. 20, no. 8, pp. 1627-1634, Aug. 2002.

[5] J. S. Xia and J. Z. Yu, "New finite-difference scheme for simulations of step-index waveguides with tilt interfaces," IEEE Photon. Technol. Lett., vol. 15, no. 9, pp. 1237-1239, Sep. 2003.

[6] J. S. Xia and J. Z. Yu, "Single-mode condition for silicon rib waveguides with trapezoidal cross-section," Opt. Commun., vol. 230, no. 4-6, pp. 253-257, 2004.

[7] J. Yamauchi, S. Harada, S. Kobori, and H. Nakano, "Modified finitedifference formula for the analysis of a step-index waveguide with a tilted interface," in Proc. Integ. Photon. Nanophoton. Res. Appl., Honolulu, HI, Jul. 2009, Paper IMG6.

[8] J. Yamauchi, T. Murata, and H. Nakano, "Analysis of rib waveguides by a fourth-order accurate finite-difference beam-propagation method," IEICE Trans., vol. J85-C, no. 4, pp. 241-248, Apr. 2002.

[9] C. Vassallo, "1993-1995 optical mode solvers," Opt. Quantum Electron., vol. 29, no. 2, pp. 95-114, Feb. 1997.

[10] J. P. Berenger, "A perfectly matched layer for the absorption of electromagnetic waves," J. Comput. Phys., vol. 114, no. 2, pp. 185-200, Oct. 1994.

[11] J. Yamauchi, S. Harada, and H. Nakano, "Minimum loss condition of a bent rectangular hollow waveguide," Microw. Opt. Technol. Lett., vol. 51, no. 12, Dec. 2009 